\title{
Silencing cuticular pigmentation genes enables RNA FISH in intact chemosensory appendages
}

Stefan Pentzold ${ }^{1 *}$, Veit Grabe ${ }^{2}$, Andrei Ogonkov ${ }^{1}$, Lydia Schmidt ${ }^{1}$, Wilhelm Boland ${ }^{1}$ and Antje Burse ${ }^{1}$

Max Planck Institute for Chemical Ecology, 'Department of Bioorganic Chemistry, ${ }^{2}$ Department of Evolutionary Neuroethology, Hans-Knöll-Str. 8, D-07745 Jena, Germany

*author to whom correspondence and material requests should be addressed, email: spentzold@ice.mpg.de

\begin{abstract}
Optical imaging of gene expression by RNA-fluorescent in situ hybridisation (FISH) in whole-mount sensory appendages of insects is often impeded by their highly pigmented cuticle. Since most chemical bleaching agents are incompatible with imaging fluorescentlabelled nucleotides, we developed a RNA interference-based method for clearing cuticular pigmentation that allows imaging of fluorescent mRNA in whole-mount appendages of insects. Silencing key genes of the tyrosine-derived pigmentation pathway by injecting dsRNA of laccase 2 or tyrosine hydroxylase in two leaf beetles species (Chrysomela populi, Phaedon cochleariae) resulted in clearance of the highly pigmented cuticle and in significant decreased light absorbance. Intact chemosensory appendages (palps, antennae and legs) from RNAi-cleared individuals were used to image expression and spatial distribution of antisense mRNA of two chemosensory genes (gustatory receptor, odorant-binding protein) via RNA FISH and confocal laser scanning microscopy. Imaging of these genes did neither work for RNAi-controls (dsGfp) due to retained pigmentation, nor for FISH-controls using sense mRNA. Furthermore, we show that several chemical bleaching agents are not feasible with FISH, either due to significant degradation of polynucleotides, lack of clearing efficacy or long incubation times. Overall, silencing pigmentation genes is a significant improvement over bleaching agents allowing fluorescence imaging in whole-mount appendages and organs.
\end{abstract}




\section{$\underline{\text { Main text }}$}

Optical imaging in combination with in situ fluorescent labelling is a powerful method to elucidate spatiotemporal patterns of gene expression and to identify cellular circuits in biological systems ${ }^{1}$. For optimal image sharpness and resolution in fluorescence microscopy, samples should ideally be transparent. However, most tissues or appendages appear opaque and may be coloured by pigments. These obstacles prevent imaging at high depth - or any optical penetration at all - into tissue due to light absorption and scattering, respectively ${ }^{2}$. Thus, sharp imaging, especially deep into a tissue volume such as from whole-mount preparations, becomes strongly limited. Alternatively, physical serial sectioning in ultra-thin slices may be performed; however, correcting single slices for alignment, geometric distortion and staining variation is time-demanding and can be error-prone ${ }^{3}$. Non-sectioning approaches such as optical sectioning by confocal laser scanning (cLSM) or light sheet microscopy, largely preserve the innate 3-dimensional structure of biological tissues and organs, but require transparent samples ${ }^{4}$. Although a diverse set of novel tissue clearing techniques has been developed for some vertebrate species to achieve whole-organ or whole-body transparency enabling imaging of fluorescent labelled molecules deep into large tissue volume $^{4-12}$, all current clearing methods come with specific limitations, e.g. they may affect structural and molecular integrity due to shrinkage or swelling of cells and tissue, quenching of fluorescence and/or altered antigenicity, among other issues ${ }^{4,8}$.

In many insect species, optical imaging is especially challenging, since pigmentation prevents fluorescence-based microscopy beneath the cuticle. Pigmentation and thus coloration can vary from colourless to yellowish or brownish to black depending on the amounts and types of melanin-like pigments incorporated ${ }^{13}$. Such pigments absorb light which results in decreased laser intensity and low optical quality as one penetrates deeper into tissue ${ }^{3,14}$. This bottleneck hampers deep imaging of intact tissue, appendages or whole bodies. Physical removal of the cuticle is less desirable due to inevitable destruction of surrounding tissue, especially in small specimen. Chemical clearing agents such as hydrogen peroxide $\left(\mathrm{H}_{2} \mathrm{O}_{2}\right)$ bleach soft tissues such as brains, which allow reasonable fluorescent imaging of receptor neuron proteins by cLSM, but it results in tissue shrinkage such as in ants ${ }^{15}$ or spherically expanded ruptures in hawkmoths ${ }^{14}$. Other bleaching protocols for whole insects with brownish bodies require at least three weeks of incubation ${ }^{16,17}$. Since many insect species such as beetles often possess a particular thick, hard and pigmented cuticle, here we present a novel methodological strategy that combines both specific and effective clearing of cuticular 
pigmentation as well as preservation of molecular and cellular integrity. This approach allows optical imaging of fluorescence-labelled nucleotides in intact appendages or other wholemount organs.

We take advantage of the proven functional importance of the enzymes tyrosine hydroxylase (TH) and laccase2 (Lac2, a phenol oxidase) in the beetle's cuticular pigmentation pathway catalysing the first and following enzymatic steps ${ }^{18,19}$ (Fig. 1a). As expected and validated by qRT-PCR, lac 2 and $T H$ were most expressed in the adult stage of the poplar leaf beetle (Chrysomela populi), especially in chemosensory appendages such as legs and antennae, which corresponds to the highest degree of pigmentation compared to other developmental stages and tissues, respectively (Fig. S1a). Silencing $T H$ and Lac2 by RNA interference (RNAi), i.e. injecting 80 or 140 ng double-stranded RNA (dsRNA) of Lac2 or TH per individual, resulted in significant clearing of cuticular pigmentation in comparison to RNAi-controls (injection of dsGfp) in adult $C$. populi as well as the mustard leaf beetle Phaedon cochleariae (Fig. 1b). Injection of dsLac2 or dsTH in young pupae reduced pigmentation already in late pupae, but not in controls (Fig. S2). Hatching rate was similar among dsLac2 (68,5\%, N=46), (dsTH 77,8\%, N=22) and dsGfp-control $(65,8 \%, \mathrm{~N}=51)$ injected individuals; RNAi adults survived at least 8 days. Importantly, RNAi-based clearing was induced in chemosensory appendages such as antennae, legs and palps (Fig. 1b, c). Transcript levels were silenced significantly, i.e. by $94,5 \%$ and $98,2 \%$ for CpopLac2 and CрорTH respectively in comparison to RNAi-controls, as validated by $\mathrm{qRT}-\mathrm{PCR}(\mathrm{P}=0.008$, $\mathrm{P}=0.016$; Fig. 1d).

To quantify clearing of cuticular pigmentation the visible spectrum of the supernatant of acid-macerated legs was measured by UV-Vis spectroscopy. The significant higher absorbance in the $400-475 \mathrm{~nm}$ region in RNAi-control samples, which is characteristic of dark-coloured products such as quinones from insect cuticles ${ }^{13}$, was missing in lesspigmented lac2- and TH-RNAi samples (Fig. 1e) showing a mean reduction in light absorbance of 78,0 and 91,4\%, respectively. Therefore, we tested whether RNAi-cleared specimens are suitable for whole-mount RNA FISH and optical imaging by cLSM without chemical bleaching that would likely degrade mRNA.

As FISH targets, two distinct chemosensory gene families were chosen: a gustatory receptor (CpopGR1) and an odorant-binding protein (CpopOBP13). Both genes were most expressed in body appendages such the head (including palps), legs and antennae as shown by qRT-PCR (Fig. S1b). Similar to other gene expression studies in beetles ${ }^{20-22}$, CpopGRl had relative low overall expression levels, even in chemosensory organs $(\sim 10 \%$ of reference 
genes), whereas OBPs such as CрорОВP13 are generally higher expressed as shown for the chemosensory appendages of $C$. populi (up to 15 times higher than reference genes). The RNAi treatment did not affect RNA FISH target sequences, i.e. the expression levels of $G R 1$ and $O B P 13$ did not differ between RNAi individuals (dsLac2 and dsTH) compared to RNAicontrols $(\mathrm{P}=0,39$ for CpopGR1; $\mathrm{P}=0,73$ for CpopOBP13, t-test). For RNA FISH, freshly dissected whole-mount chemosensory appendages (palps, antennae and legs) from RNAiLac2 and RNAi-TH cleared beetles were incubated with biotin-labelled antisense mRNA (or sense mRNA as control) targeting either CpopGR1 or CpopOBP13 and combined with digoxigenin-labelled CpopActin as housekeeping gene. Importantly, gene expression of CpopGR1 and CPopOBP13 in intact appendages was successfully imaged via cLSM in those individuals that were silenced in Lac2 or TH (Fig. 2a, Fig. S3a, b). For example, cells expressing CpopGRI were mainly proximal in the palp (Fig. 2a, i) and overall less abundant than those expressing CpopOBP13 showing a broader distribution (Fig. 2a, ii). This is similar to the expression measured via qRT-PCR indicating relatively low or high expression for CpopGR1 or CpopOBP13, respectively (Fig. S1b). Furthermore, in the antennae, CpopOBP13 was expressed in the proximal and more distal parts (Fig. S3a), whereas in the tibia CрорОВP13 was mainly expressed in proximal cells (Fig. S3b). Cuticular autofluorescence in RNAi-cleared organs remains to some extant after optical sectioning by cLSM (e.g. Fig. 2a i, ii), which is most likely caused by chitin ${ }^{23}$. In another study, $\mathrm{H}_{2} \mathrm{O}_{2}$-based quenching of autofluorescence was used to image microbial endosymbionts living in inner insect tissues such as the gut ${ }^{16}$; in general, many tissue clearing methods do not necessarily remove background autofluorescence ${ }^{2}$. In contrast to RNAi-cleared specimens, optical imaging of CpopGR1 and CpopOBP13 was not possible in RNAi-controls (dsGfp) due to retained pigmentation that prevented optical penetration (Fig. 2a, iii). As further controls, using sense mRNA for FISH did not result in specific fluorescence for GR1 or OBP13 in any of the RNAi-treated beetles (ds $L a c 2$, dsTH or dsGfp-controls) (Fig. 2a, Fig. S3c). Nuclear DNA staining via fluorescent DAPI confirms cell integrity in RNAi-cleared tarsi of $C$. populi and $P$. cochleariae (Fig. 2b).

To corroborate that RNAi-based clearing of cuticular pigmentation is the means of choice for using whole-mount organs in RNA FISH, we show that several bleaching chemicals are not feasible FISH, either due to significant degradation of polynucleotides, lack of clearing efficacy or long incubation times. We first tested $\mathrm{H}_{2} \mathrm{O}_{2}$ of which hydroxyl radicals and $\mathrm{OOH}$-groups are known to destroy the cuticle-pigment melanin ${ }^{24}$. Whereas incubation of dissected body appendages in $35 \% \mathrm{H}_{2} \mathrm{O}_{2}$ for 3 days reduced cuticular pigmentation in legs 
(Fig. 2c), it degraded $99,2 \%$ of the total RNA as measured by qRT-PCR using two housekeeping genes in comparison to water-incubated controls (Fig. 2d); similarly, antennae were chemically bleached resulting in degradation of 91,6\% total RNA (Fig. 2c, d). Incubation of antennae and legs for 3 days in water (Fig. 2c) or $10 \% \mathrm{H}_{2} \mathrm{O}_{2}$ did not reduce cuticular pigmentation, but degraded RNA (Fig. 2d). When incubating in alcoholic $6 \% \mathrm{H}_{2} \mathrm{O}_{2}$, it took at least three weeks until bleaching of intact or bipartite antennae and legs (Fig. S4a). This is similarly long to bleaching brown-coloured, but relatively small insects such as lice, bat flies or aphids ${ }^{16}$. However, almost two third of total RNA $(63,7 \%)$ was degraded despite prior to tissue fixation. When whole beetles were incubated in $35 \% \mathrm{H}_{2} \mathrm{O}_{2}$ for five days, cuticular decolouration was evident, but partly incomplete, especially on the legs and wings that were also damaged (Fig. S4b). Consequently, cell integrity in legs and antennae was not retained in $\mathrm{H}_{2} \mathrm{O}_{2}$-bleached samples as indicated by unsuccessful DAPI staining (Fig. 2e). The celldamaging characteristics of $\mathrm{H}_{2} \mathrm{O}_{2}$ resulting in degradation of total RNA and decreased fluorescence emission from green fluorescent proteins ${ }^{25}$, makes this bleaching method unsuitable for (RNA) FISH. This is especially important when targeting lowly expressed genes such as most insect GRs ${ }^{20}$. Other clearing agents such as methyl salicylate ${ }^{3}$ did clear soft tissues such as the brain of $C$. populi, after dehydration in an ethanol series, but not cuticular pigmentation (Fig. S5a). Similarly, a mixture of benzyl alcohol/benzyl benzoate did not clear cuticular pigmentation in antennae or legs (Fig. S5b).

RNAi-based clearing of cuticular pigmentation is a significant improvement compared to chemical bleaching agents, since it preserves molecular and cellular integrity and enables optical imaging of fluorescent-labelled nucleotides deep into tissue of intact whole-mount samples. This makes serial sectioning of organs redundant. We expect that our method also works with other, non-chemosensory genes regardless of whether they encode soluble proteins or membrane receptors, or whether they are lowly or highly expressed. Moreover, since RNAi-based suppression of $L a c 2$ or $T H$ decreases cuticular pigmentation in different coleopteran $^{18,26-28}$ and several other non-coleopteran insect species ${ }^{29-34}$, our method can be adapted to many other hexapod species enabling investigations beyond transparent embryos ${ }^{35}$ or ovaries ${ }^{36}$. Imaging beneath the RNAi-cleared cuticle may further benefit from light sheet microscopy that minimizes fluorophore bleaching and phototoxic effects. Finally, RNAibased cuticle clearing complements existing toolkits such as by enabling in vivo calcium imaging, in contrast to chemical bleaching which does not maintain most life functions. 

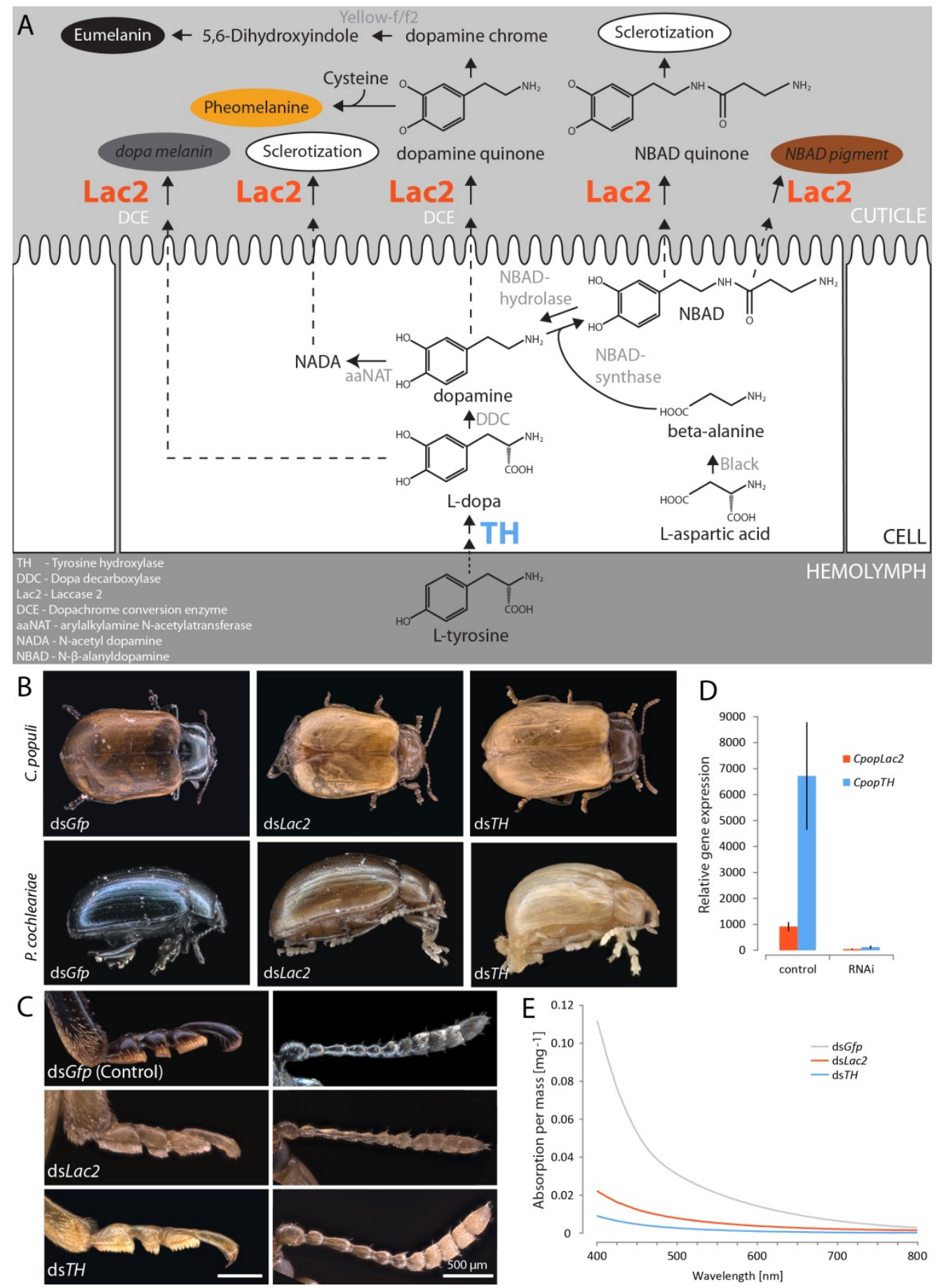

Figure 1. Silencing $T H$ and $L a c 2$ genes by RNAi reduces cuticular pigmentation in adult leaf beetles. A. Tyrosine-derived cuticular pigmentation pathway in insects illustrating the involvement of tyrosine hydroxylase (TH, blue) and Laccase2 (Lac2, orange) enzymes with their respective substrates. The resulting pigments are coloured as proposed by ${ }^{19,37,38}$. B: Phenotypes of adult $C$. populi and P. cochleariae beetles silenced in Lac2 and $T H$ by RNAi (dsRNA injection) are less pigmented and thus lighter than RNAi-controls (dsGfp injection). C: Detail on chemosensory appendages such as tarsi (left) and antennae (right) of RNAi and control individuals indicating cleared pigmentation. D: Efficacy of RNAi-mediated silencing of CpopLac2 and CpopTH in comparison to RNAi-controls (injection of dsGfp) as analysed by qRT-PCR. Differences in relative expression are statistically significant between the two treatments (Lac2: $\mathrm{P}=0,008 ;$;H: $\mathrm{P}=0,016$; Wilcoxon-Rank-Sum test) and refer to $94,5 \%$ and $98,2 \%$ respectively, transcript silencing in comparison to RNAi-controls. Bars represent \pm s.e.m. E: Visible spectral changes measured by UV-Vis spectroscopy are associated with silencing Lac2 or $T H$ by RNAi in comparison to RNAi-controls (ds $G f p$ ). Mean reduction in absorbance was $78 \%$ for ds $L a c 2$ and $91,4 \%$ for $\mathrm{ds} T H$. Samples were macerated in acid and absorbance of the supernatant was measured from $400-800 \mathrm{~nm}$. 


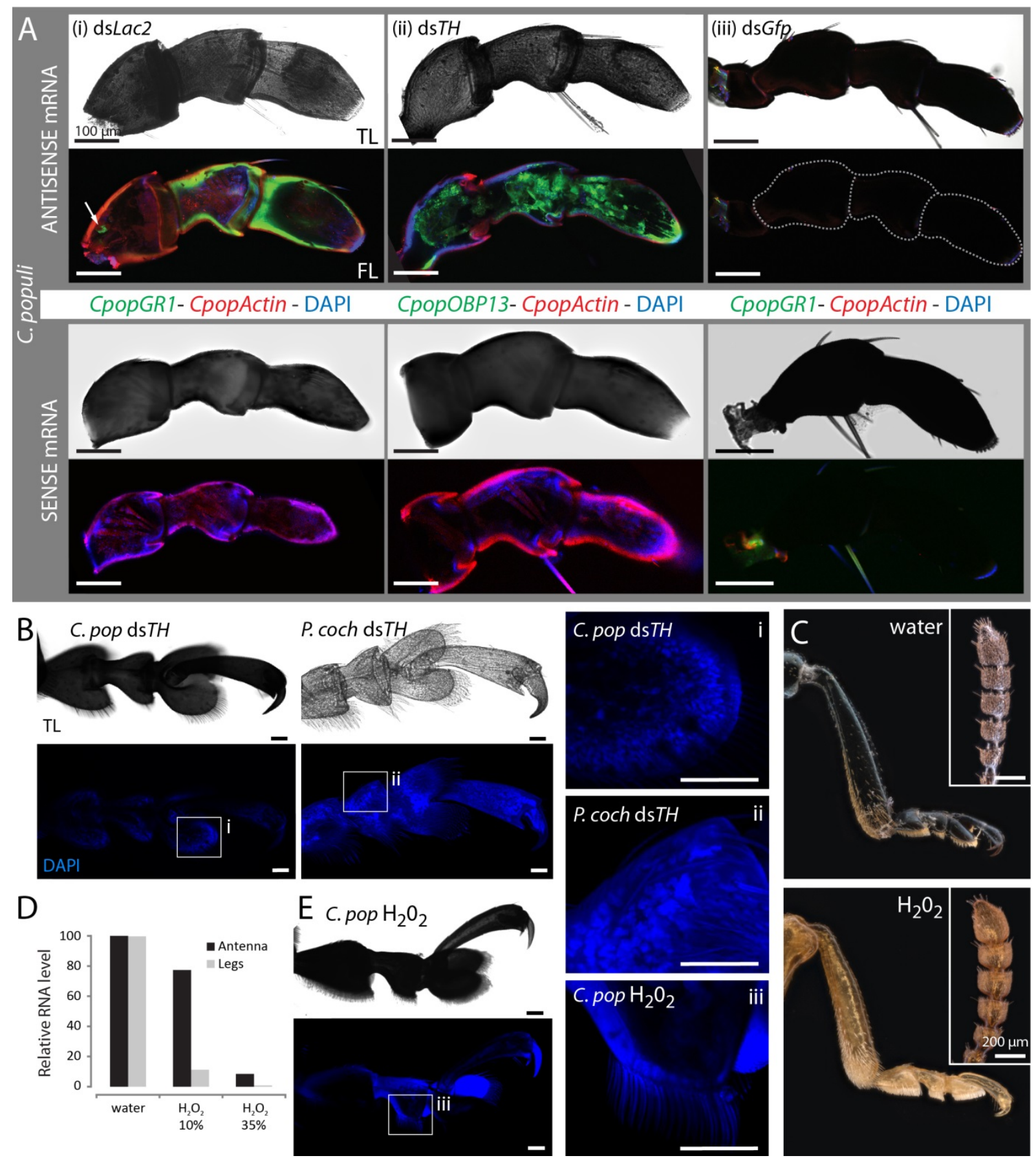

Figure 2. Specific clearing of cuticular pigmentation by RNAi enables RNA FISH in whole-mount appendages of leaf beetles. A: Maxillary palps from (i) $L a c 2$ or (ii) $T H$-silenced individuals with biotinlabelled mRNA of either a gustatory receptor (CpopGR1) or an odorant-binding protein (CpopOBP13) (both green) in combination with digoxigenin-labelled mRNA for CpopActin (red) as well as nuclear staining via DAPI (blue fluorescence). Due to remained pigmentation in RNAi-controls (dsGfp, iii) RNA FISH using the same mRNA probes was not possible. Whereas antisense mRNA binds to the target sequence (upper panels), sense mRNA of GR1 or OBP13 as control does not bind (lower panels). TL transmitted light microscopy (light panels), FL - fluorescence image from confocal laser scanning microscopy (dark panels). B: Tarsi of $C$. populi and $P$. cochleariae cleared by silencing $T H$ and stained with DAPI targeting nuclear dsDNA confirms cellular integrity of RNAi treatments (i, ii). C: Incubation of antennae and legs of $C$. populi in $\mathrm{H}_{2} \mathrm{O}_{2}(35 \%)$ for 3 days clears cuticular pigmentation in comparison to water-controls, but D: results in significant degradation of total RNA in both organs. E: Tarsi incubated in $\mathrm{H}_{2} \mathrm{O}_{2}$ for 3 days could not be stained by DAPI indicating cellular damage by $\mathrm{H}_{2} \mathrm{O}_{2}$. Fluorescence is cuticular autofluorescence (iii). 


\section{Methods}

\section{Specimen, RNA extraction and cDNA synthesis}

Poplar leaf beetles (C. populi, L.,) were collected close to Dornburg (+51 $\left.00^{\prime} 52^{\prime \prime},+11^{\circ} 38^{\prime} 17^{\prime \prime}\right)$ in Thuringia, Germany, where beetles were feeding on Populus maximowiczii x Populus nigra and reared in a climate chamber provided with fresh $P$. nigra twigs under 16:8 h L:D cycles at $20^{\circ} \mathrm{C}$. Mustard leaf beetles (P. cochleariae, F.) were lab-reared on Brassica oleracea under same conditions. Both beetle species belong to the tribe Chrysomelini within the family Chrysomelidae. After homogenizing adult beetles using mortar and pestle in liquid nitrogen. RNA was purified using RNAqueous ${ }^{\mathrm{TM}}$ Total RNA Isolation Kit (Ambion) including DNase treatment following the manufacturer's instruction. RNA concentration was measured on a NanoDrop ${ }^{\text {TM }}$ One (Thermo Scientific). Synthesis of cDNA was carried out using SuperScript III Reverse Transcriptase and oligo(dT) ${ }_{20}$ (Invitrogen) following manufacturer's protocol.

\section{Identification of candidate genes and molecular cloning}

Orthologues of Lac2 and TH in C. populi and P. cochleariae were identified by performing a BLAST search against the respective transcriptome ${ }^{39,40}$ using nucleotide sequences of Lac2 (GenBank accession number: AY884061.2) and TH (EF592178) from Tribolium castaneum as template ${ }^{18,28}$. As targets for RNA FISH, a gustatory receptor (CpopGRl) and an odorantbinding protein (CpopOBP13) were identified from the transcriptome of C. populi ${ }^{39}$ by BLAST search using the template sequences CbowGR1 (GenBank: KT381521) and CbowOBP13 (KT381495) from the cabbage beetle Colaphellus bowringi ${ }^{22}$ (Chrysomelini, Chrysomelidae). The sequence of the house-keeping gene actin from C. populi was obtained from GenBank (JX122919.1). Gene specific primers were designed using Primer3 software (see table S1); a T7 promoter sequence (5'-TAATACGACTCACTATAGGGAGA-3') was appended to forward and reverse primers of Lac2 (CpopLac2, PcoLac2) and TH (CpopTH, $P c o T H)$ to allow in vitro synthesis of dsRNA for RNA interference (see below). Therefore, PCR amplification was carried out using a proof-reading DNA polymerase (Phusion HighFidelity, ThermoFisher). Amplicons were analysed via gel electrophoresis, purified using QIAquick PCR Purification (Qiagen) and sequenced. After RACE-PCR to verify full-length sequence using SMARTer RACE cDNA Amplification Kit (Clontech), CpopGR1, CpopOBP13 and CpopActin were PCR-amplified without modification, sequenced and ligated into pCR-BluntII-TOPO (Invitrogen) posseesing opposing T7 and SP6 promoter sequences to allow in vitro synthesis from one template for generation of sense and antisense probes for RNA FISH (see below: Synthesis of labelled RNA FISH probes).

\section{RNA interference}

\section{Synthesis of dsRNA}

Synthesis of dsRNA was carried out using MEGAscript ${ }^{\circledR}$ RNAi Kit (Ambion) according to the manufacturer's recommendations. In brief, PCR product with opposing T7 promoters flanking the target sequences ( $\mathrm{Lac} 2, \mathrm{TH}$ from $\mathrm{C}$. populi or P. cochleariae) was transcribed in vitro by incubating for $6 \mathrm{~h}$ at $37^{\circ} \mathrm{C}$ with T7-Polymerase, ATP, CTP, GTP and UTP including RNase inhibitor protein. Formation of dsRNA was induced by incubating the reaction mixture for $5 \mathrm{~min}$ at $75^{\circ} \mathrm{C}$ and then cooling to room temperature (RT). After nuclease digestion of 
single-stranded RNA and DNA, dsRNA was purified using filter cartridges and centrifugation. Finally, dsRNA was ethanol precipitated, resuspended in $0.9 \%$ physiological $\mathrm{NaCl}$ and adjusted to $1 \mu \mathrm{g} / \mu \mathrm{l}$. The concentration and integrity of the dsRNA were determined by spectrometry (NanoDrop One, Thermo Scientific) and gel electrophoresis. The following lengths of dsRNA were generated: CpopdsTH: 535 bp, CpopdsLac2: 548 bp, PcodsLac2: 521 bp, PcodsTH: 544 bp (all excluding 23bp T7-promoter sequence, see table S1). DsRNA of Gfp (719 bp) was used as control.

\section{$\underline{\text { Microinjection }}$}

Microinjections were performed using pulled borosilicate glass capillaries and Nanoliter 2000 injector (World precision instruments, Inc.) Injection was parasagittally between the pro- and mesothorax of either last larval instars or 3-day old pupae of $C$. populi and P. cochleariae. 12 biological replicates were run, i.e. a total of 48 . Insects were briefly anesthetized on ice before and during injection. Each specimen was injected 20, 70 or $150 \mathrm{ng}$, respectively of total dsRNA, which corresponds to two times 9.2, 32.2 or $69.0 \mathrm{nl}$ of Lac2, TH, lac2+TH or Gfp. Five to ten days after eclosure and rearing adult beetles on $P$. nigra, chemosensory appendages such as legs, antennae and palps were dissected for use in RNA FISH (see below). Additionally, efficacy of RNAi-mediated knockdown of CpopLac2 and CpopTH was assessed using quantitative real-time PCR on cDNA derived from legs. Therefore, transcript abundance of $L a c 2$ or $T H$ in dsGfp control samples was set as baseline (100\%) and compared to their expression in RNAi beetles. For statistical analysis, mean ct values of Lac2 or TH from both groups (dsGfp control versus RNAi) were compared for significant differences using Wilcoxon Rank-Sum test (SigmaPlot 12.0). Five biological replicates were analysed from both groups. For primers see table S1.

\section{Quantitative real time-PCR}

Using qRT-PCR transcript abundance of (i) Lac2 and TH was analysed in C. populi in different developmental stages such as larvae, pupae and adults, and in adult legs, antennae and wings (three to four biological replicates), (ii) CpopGRI and CpopOBP13 in mentioned adult tissues such as gut, wings, thorax (excluding head and legs), legs, antennae and head including palps (six biological replicates). Data were quantified relative to the mRNA levels of the reference genes eukaryotic elongation factor 1-alpha (EF1a) and initiation factor 4A (eIF4A) using the $2-{ }^{\Delta \Delta \mathrm{Ct}}-$ method $^{41}$. Data were acquired on a CFX-96 Touch $^{\mathrm{TM}}$ Real-Time PCR Detection System (Bio-Rad) using Brilliant III Ultra-Fast SYBR ${ }^{\circ}$ Green QPCR Master Mix (Agilent Technologies) and cDNA as template. Distilled water as template served as negative control. Two technical replicates were analysed; those with a $\mathrm{Ct}$ difference of $>0.5$ were repeated. To exclude effects of RNAi (dsLac2, dsGfp) on the expression level of CpopGRl and CpopOBP13 (as RNA FISH targets, see below), their expression was compared to ds $L a c 2$ or ds $G f p$-injected individuals by qRT-PCR on cDNA derived from of legs (five biological replicates per group). Statistical difference of the ${ }^{\Delta \mathrm{Ct}}$-values between the two groups was compared using t-test (SigmaPlot 12.0). Standard curves in five different 10fold dilutions using plasmids containing the target gene were used to calculate amplification efficiency. For each primer pair sigmoidal amplification curves were obtained with single 
melt curve peaks. No signals were detected for the negative controls. For primers used see table S1.

\section{Whole-mount double RNA FISH}

$\underline{\text { Synthesis of labelled RNA FISH probes }}$

Plasmids containing target sequences (CPopGR1, CpopOBP13, CpopActin) flanked by opposing T7 and SP6 promoter sequences were linearized using NotI or BamHI. Subsequent in vitro transcription of labelled antisense or sense RNA was carried out using SP6 or T7 polymerases in the presence of biotin (GR1, OBP13) or digoxigenin (DIG; actin) following manufacturer's procedure (RNA Labelling mix 10x, Roche). After incubation for $3 \mathrm{~h}$ at $37^{\circ} \mathrm{C}$, RNA was precipitated using ethanol, dissolved in RNase-free water and diluted in hybridisation buffer $(50 \%$ deionized formamide, $2 \times \mathrm{SSC}, 0.2 \mathrm{mg} / \mathrm{mL}$ sonicated herring sperm DNA, $200 \mu \mathrm{g} / \mathrm{ml}$ yeast tRNA, and 10\% dextran sulfate, water bidest). The following lengths were generated: CpopGR1: $390 \mathrm{nt}$ and CpopOBP13: $513 \mathrm{nt}$ (both biotin-labelled) and CpopActin (DIG-labelled) about $450 \mathrm{nt}$ from original $1131 \mathrm{nt}$ after incubation in hydrolysis buffer $\left(80 \mathrm{mM} \mathrm{NaHCO}_{3}\right.$ and $\left.120 \mathrm{mM} \mathrm{Na}_{2} \mathrm{CO}_{3}\right)$ at $60^{\circ} \mathrm{C}$ for $14,5 \mathrm{~min}$ (formula see ${ }^{36}$ ).

\section{Fixation and FISH procedure}

Intact and whole palps (labial, maxillary), antennae and legs were freshly dissected from icechilled adult $C$. populi beetles. To improve penetration of labelled FISH probes, antennae and legs were cross-sectioned into a distal and proximal part (at the $5^{\text {th }}$ flagellomere and between tibia and femur, respectively). Samples were fixed in $4 \%$ paraformaldehyde in $1 \mathrm{M} \mathrm{NaHCO}_{3}$ at $4^{\circ} \mathrm{C}$ for $24 \mathrm{~h}$. Organs were washed in 1xPBS containing $0,03 \%$ TritonX100 for $1 \mathrm{~min}$ at RT and incubated in $0.2 \mathrm{M} \mathrm{HCl}$ containing $0,03 \%$ TritonX100 for $10 \mathrm{~min}$. After washing in 1xPBS with $1 \%$ TritonX100 for $1 \mathrm{~min}$ at $\mathrm{RT}$, prehybridization was carried out by incubating samples in hybridisation buffer for $4 \mathrm{~h}$ at $4^{\circ} \mathrm{C}$ and for additional $6 \mathrm{~h}$ at $55^{\circ} \mathrm{C}$. Hybridisation of labelled RNA probes to endogenous transcripts was performed at $55^{\circ} \mathrm{C}$ for 3 days. The following combinations were used: antisense or sense mRNA of GRl or OBP13 (biotinlabelled) in combination with antisense of actin (DIG-labelled). After washing in $0.1 \mathrm{xSSC}$ with $0,03 \%$ TritonX100 four times at $60^{\circ} \mathrm{C}$ by horizontal shaking at $350 \mathrm{rpm}$, samples were blocked in 1\%-blocking solution (Roche) diluted in TBS and 0,03\% TritonX100 for $6 \mathrm{~h}$ at $4^{\circ} \mathrm{C}$. Detection of DIG-labelled probes was achieved by using an anti-DIG-conjugated alkaline phosphatase that dephosphorylates non-fluorochromic HNPP added to the sample into HNP that precipitates to RNA in the presence of Fast Red TR (HNPP Fluorescent Detection Set, Roche). Detection of biotin-labelled probes was achieved by streptavidincoupled-horse radish peroxidase and tyramide signal amplification TSA fluorescein detection kit (Perkin Elmer NEL701A001KT). In both cases, antibody incubation was carried out for 3 days at $4^{\circ} \mathrm{C}$ followed by substrate incubation for $6 \mathrm{~h}$. Intermediate washing steps were carried out three times for $10 \mathrm{~min}$ in TBS- $0,05 \%$ Tween. Counterstaining of cellular dsDNA was done using DAPI (4',6-diamidino-2-phenylindole ) nucleic acid stain (Molecular probes) in 30 $\mathrm{mM}$ PBS and incubated for $30 \mathrm{~min}$ at RT in dark and three times washing in PBS. Finally samples were transferred onto microscope slides covered in Vectashield antifade mounting medium (Vector Laboratories).

\section{Microscopy and image processing}


Fluorescent images were acquired with a ZEISS LSM 880 Confocal Laser Scanning Microscope using a 20x/0.8 Plan-Apochromat or 40x/1.2 C-Apochromat W (all ZEISS, Germany), respectively. Excitation of the fluorophores was conducted through a $405 \mathrm{~nm}$ laser diode, a $488 \mathrm{~nm}$ Argon laser and a $543 \mathrm{~nm}$ Helium-Neon laser (ZEISS, Germany). The systems spectral Quasar detector was setup to detect the fluorophores at 415-490 nm for DAPI, 490-561 nm for biotin-labelled probes and 551-735 nm for DIG-labelled probes. The pinhole was adjusted to 1 airy unit for the crucial channel of the in situ probe. Reflected light brightfield images from the whole beetles as well as their appendages were obtained on an AXIO Zoom V.16 (ZEISS, Germany). Images were processed using the following imaging software: ZEISS ZEN, Helicon Focus, Fiji and Adobe Photoshop CS.

\section{Absorbance measurement}

Legs and antennae were ground in $6 \mathrm{M} \mathrm{HCl}$ and centrifuged to pellet cell and tissue debris. Supernatant was used to measure absorbance from 400 to $800 \mathrm{~nm}$ per milligram ground tissue on a UV/VIS spectrophotometer (Jasco V-550).

\section{Chemical bleaching}

Freshly dissected and fixed antennae and legs from C. populi were incubated in $\mathrm{H}_{2} \mathrm{O}_{2}$ (SigmaAldrich 18304) at different concentrations (aqueous 35.5\%, 10\%) or (alcoholic 6\%) versus water or ethanol controls at RT. In addition, whole beetles were incubated in $35.5 \% \mathrm{H}_{2} \mathrm{O}_{2}$ for 5 days at RT. For incubation in methyl salicylate freshly dissected heads of C. populi were dehydrated in an ethanol series, transferred in glass vials containing 1:1 ethanol:methyl salicylate for incubation for $1 \mathrm{~h}$ and finally substituted by $100 \%$ methyl salicylate (Sigma Aldrich M6752) for incubation for one week at RT during gentle shaking (350 rpm). After dehydrating further samples in a methanol series, they were incubated in a 1:2 mixture of BABB (Benzyl alcohol/benzyl benzoate) and incubated for three weeks.

\section{RNA degradation}

Degradation of cellular RNA was analysed from antennae and legs (incubated for 3 days in $35.5 \% \mathrm{H}_{2} \mathrm{O}_{2}$ or $6 \%$ alcoholic $\mathrm{H}_{2} \mathrm{O}_{2}$ in comparison to control incubations (water or ethanol) via qRT-PCR after RNA extraction and cDNA synthesis from three biological replicates (5-10 antennae or legs mixed) as described above. Therefore, mean ${ }^{\Delta \Delta \mathrm{Ct}}$-values of two reference genes (EFIa and IF4a) were compared between the two test and control treatments taking the latter values as baseline (100\%) of mRNA abundance.

\section{Data availability}

The data that support the findings of this study are available from the corresponding author upon reasonable request. Sequences generated in this study are deposited in GenBank (www.ncbi.nlm.nih.gov/genbank) under the following accession numbers: CpopLac2: MH253687; PcoLac2: MH253688; CpopTH: MH253689; PcoTH: MH253690; CpopGR1: MH253691; СрорОВР13: MH253692. 
Author contributions: S.P., W.B. and A.B. conceived and designed the study. S.P. performed RNA extractions, cDNA synthesis, identification of candidate genes, molecular cloning, qRT-PCR, FISH probe synthesis, fixation and RNA degradation assay. S.P. and A.O. performed chemical bleachings. V.G. carried out microscopic analyses by cLSM, processed images and constructed illustrations. A.O. analysed RNAi efficacy, performed dsRNA microinjection and FISH procedure. A.O. and A.B. analysed survival of RNAi beetles. L.S. synthesised dsRNA, did microinjections and prepared buffer solutions. S.P., A.O., V.G. and A.B. analysed the data. S.P. wrote the manuscript, all authors revised it.

Competing interests: The authors declare no competing interests.

Acknowledgements: S.P. acknowledges project funding from the European Union's Horizon 2020 research and innovation programme under the Marie Skłodowska-Curie grant agreement No 705151. All authors are grateful to Vera L. Hopfenmüller for running replicates of FISH and to Tobias Becker for setting up the absorbance measurement. Further financial support from the Max Planck Society is acknowledged. 


\section{Supplementary figures and tables}
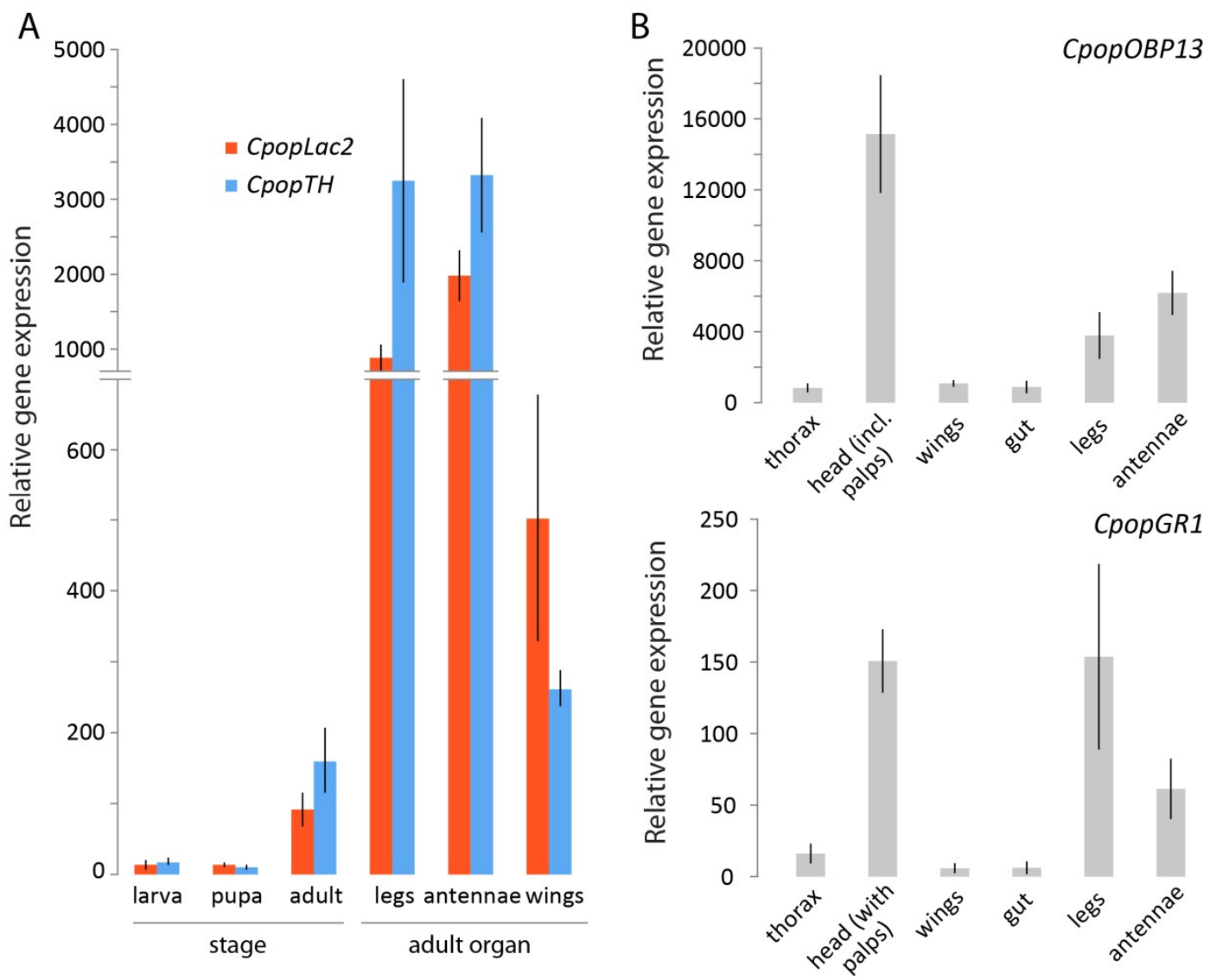

Supplementary Figure S1. A: Expression of $L a c 2$ and $T H$ as RNAi targets in untreated stages and adult tissues of C. populi as analysed by qRT-PCR. B: Expression of CpopGR1 and CpopOBP13 as RNA FISH targets in untreated tissues of adult $C$. populi as analysed by qRT-PCR. Bars represent \pm s.e.m.

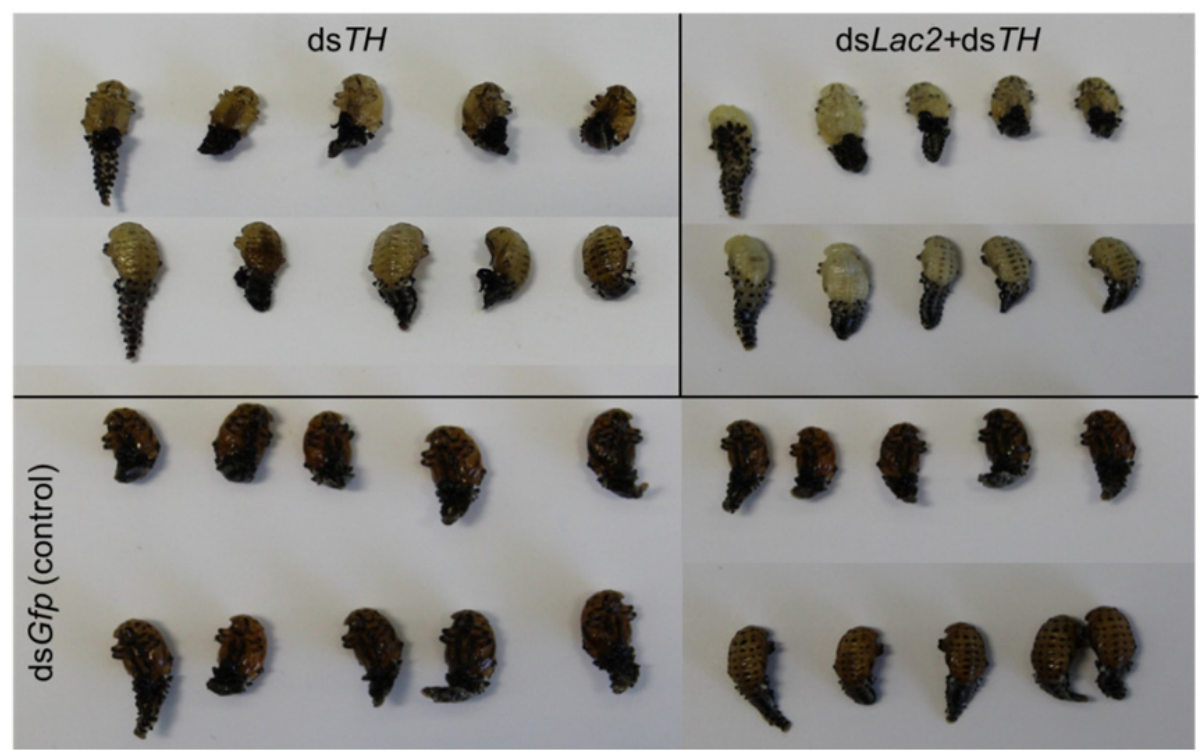

Supplementary Figure S2. Young C. populi pupae of the same age were injected dsRNA targeting $T H$ or $L a c 2+T H$ combined. RNAi-based clearing of cuticular pigmentation was evident in both cases in late pupae in comparison to darker RNAi-controls (ds $G f p$ injection). 

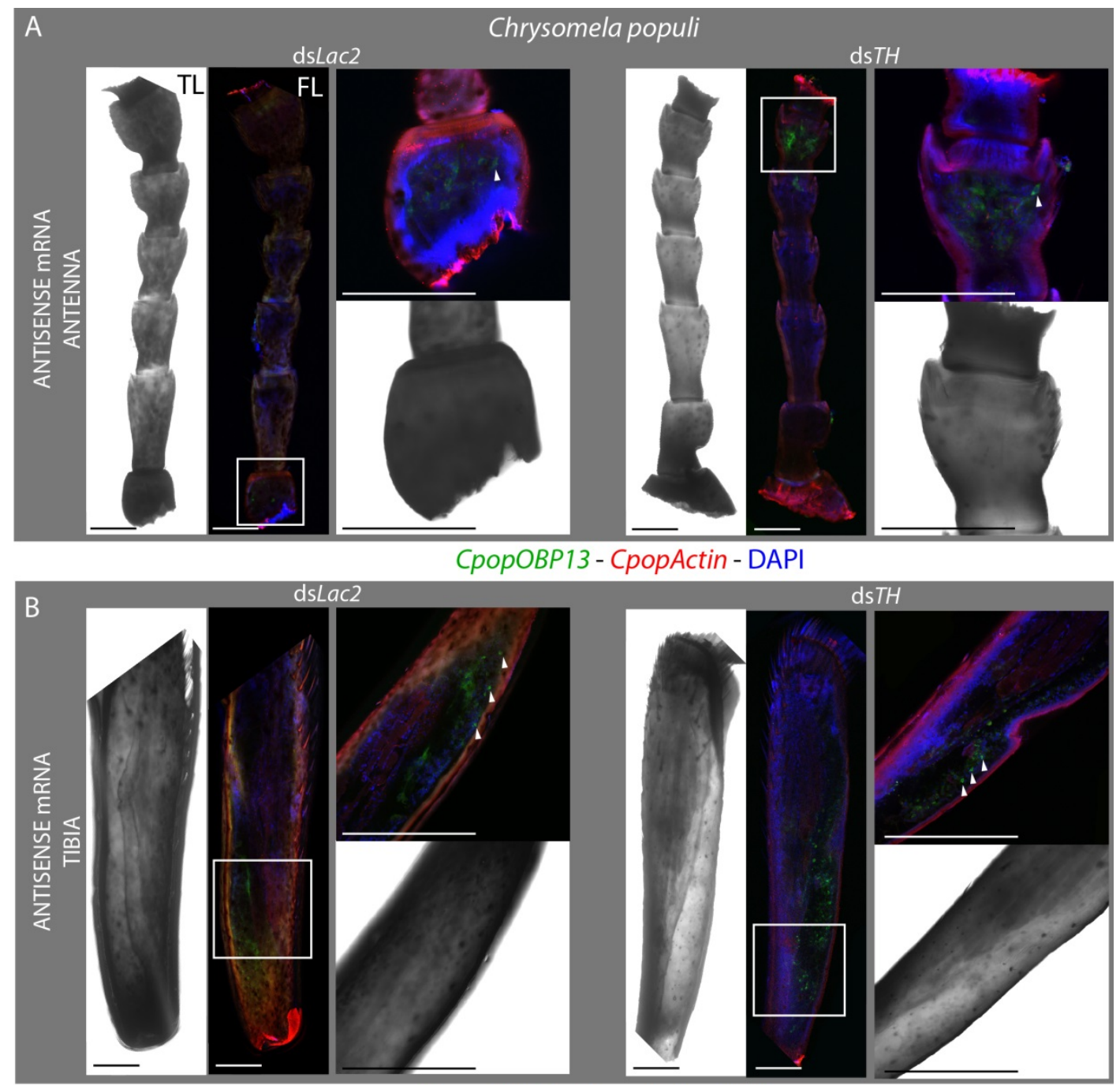

СрорОВP13 - CpopActin - DAPI

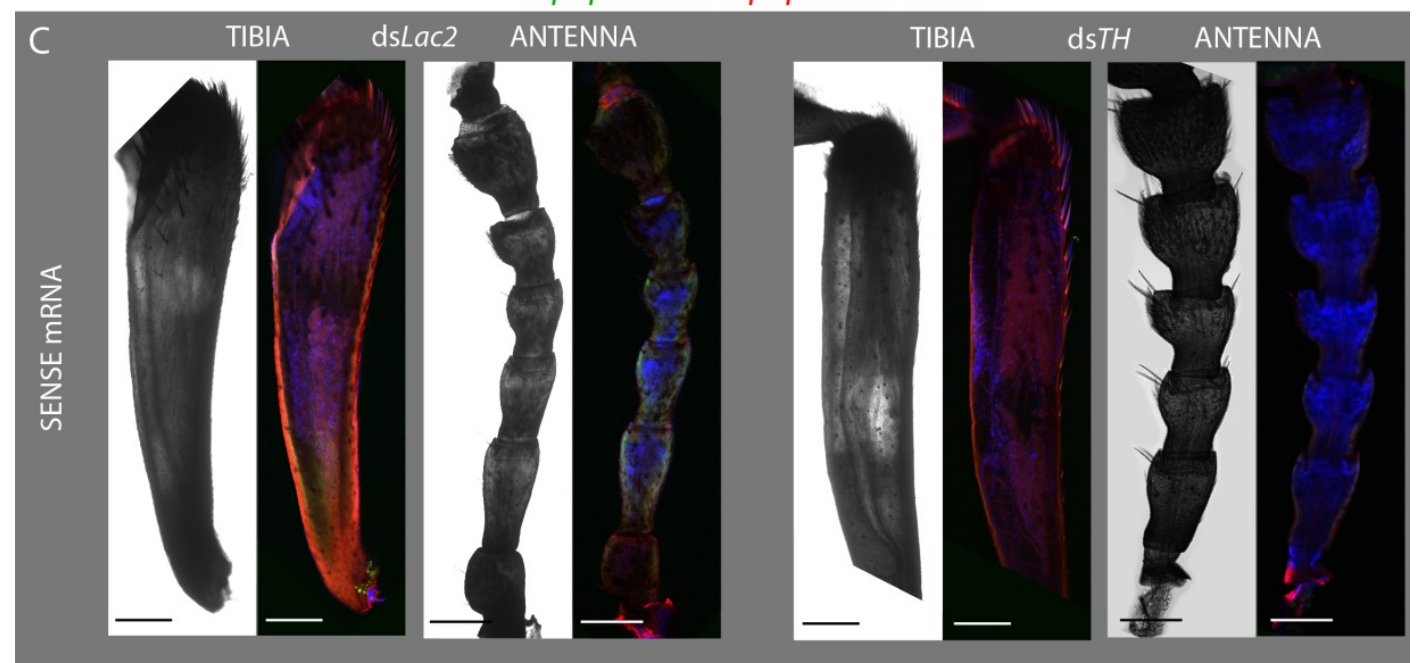

Supplementary Figure S3. Clearing of cuticular pigmentation by RNAi enables FISH in whole-mount appendages of $C$. populi. A: Antennae and B: tibia from Lac2 or $T H$-silenced individuals with biotinlabelled antisense mRNA of an odorant-binding protein (CрорОВP13) (green fluorescence) in combination with digoxigenin-labelled antisense mRNA for CpopActin (red) as well as DAPI staining nuclear dsDNA (blue). C: Using sense mRNA of $O B P 13$ as negative control in both RNAi-cleared appendages did not result in distinct green fluorescence, whereas positive controls (antisense CpopActin, DAPI) resulted in similar fluorescent signals as in A and B. Arrowheads indicate exemplary stained cells. Proximal parts of the organs are at the bottom, and distal part at the top of the image. TL - transmitted light microscopy (light panels), FL - fluorescence image from confocal laser scanning microscopy. Scale bar represents $200 \mu \mathrm{m}$. 

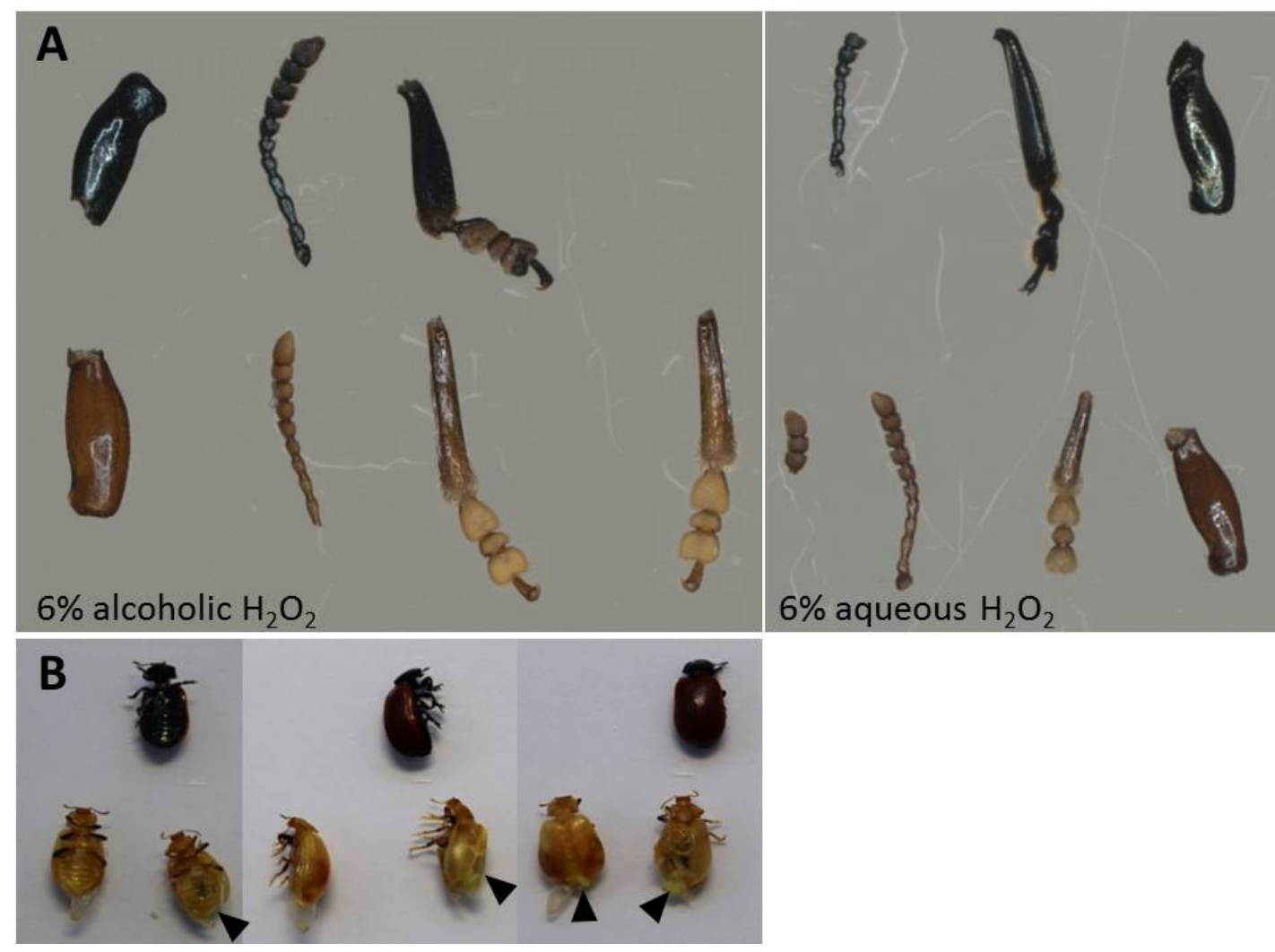

$6 \%$ aqueous $\mathrm{H}_{2} \mathrm{O}_{2}$

Supplementary Figure S4. A: Incubation of antennae and bipartite legs from $C$. populi in 6\% alcoholic or aqueous $\mathrm{H}_{2} \mathrm{O}_{2}$ for three weeks clears pigmentation in comparison to control incubations (ethanol or water), but degrades almost two third of total RNA (see text). B: Incubation of whole beetles in $35 \% \mathrm{H}_{2} \mathrm{O}_{2}$ (bottom row) for five days results in cuticular decolouration (in comparison to controls, top row), which is however partly incomplete, especially on the legs while in some cases organs such as wings were damaged (arrowheads).
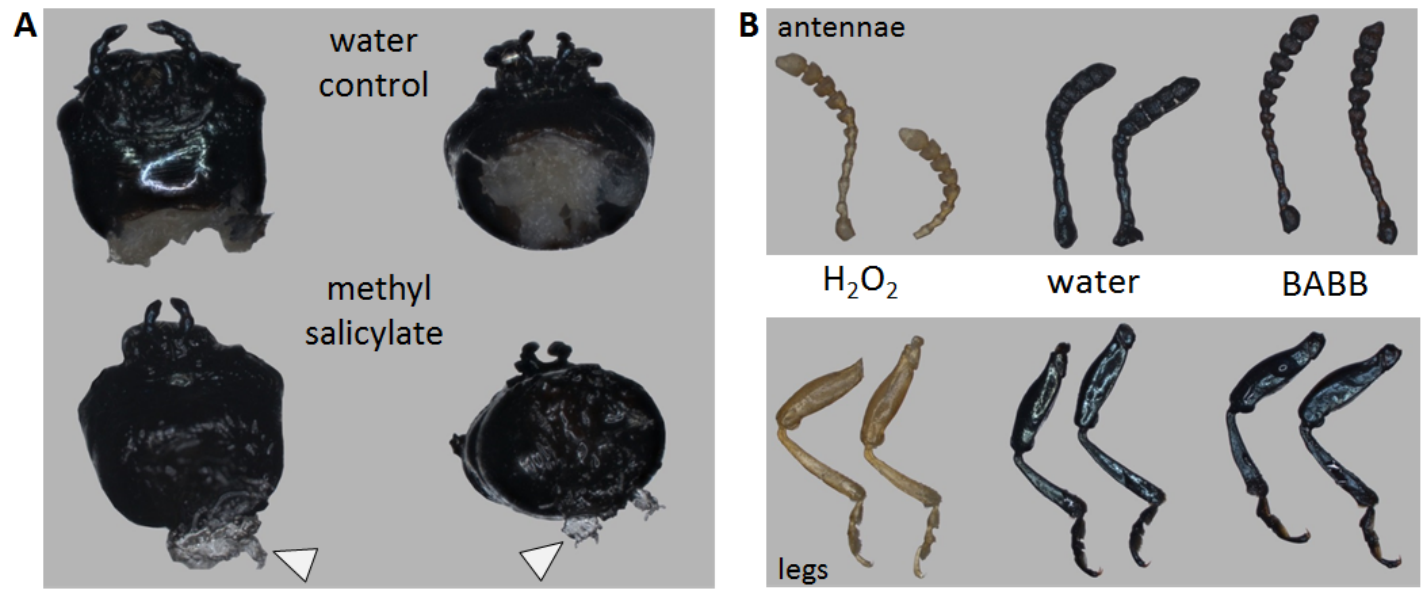

Supplementary Figure S5. Incubation of chemosensory appendages of $C$. populi in A: methyl salicylate for one week cleared soft tissue such as the brain (arrows), but not the cuticle; B: BAAB (1:2 benzyl alcohol/benzyl benzoate) for three weeks did not clear cuticular pigmentation of antennae or legs in contrast to incubation in $35 \% \mathrm{H}_{2} \mathrm{O}_{2}$. All incubations were done after fixating and dehydrating samples. 
bioRxiv preprint doi: https://doi.org/10.1101/318535; this version posted May 11,2018 . The copyright holder for this preprint (which was not certified by peer review) is the author/funder, who has granted bioRxiv a license to display the preprint in perpetuity. It is made available under aCC-BY-NC-ND 4.0 International license.

Supplementary Table S1: Primer sequences used for qRT-PCR, RNAi and RNA FISH. Cpop Chrysomela populi; Pco - Phaedon cochleariae. T7 promoter sequence in italics. eIF4A - eukaryotic initiation factor 4 alpha; EF1 - eukaryotic elongation factor 1; Lac2 - laccase2; TH - tyrosine hydroxylase; GR - gustatory receptor; OBP - odorant binding protein; fw - forward; rev - reverse.

\begin{tabular}{|l|l|}
\hline Primer name & \\
\hline qRT-PCR: & \multicolumn{1}{c|}{$\mathbf{5}^{6}-\mathbf{3}$ '-sequence } \\
\hline CpopeIF4A_fw & TTTGTAATACCCGCCGCAAG \\
CpopeIF4A_rev & TCCATGCATCGCAGAAACAG \\
\hline CpopEF1a_fw & TCATCGGTCACGTAGATTCTGG \\
CpopEF1a_rev & TTTCGATGGTACGCTTGTCG \\
\hline CpopLac2q_fw & CTCGTTTTCTGGAGAGAGATAC \\
CpopLac2q_rev & CCAATCCTCTGAGTTGTATCC \\
\hline CpopTHq_fw & CTAATGACTCCAGTGTAGAACC \\
CpopTHq_rev & GACGACTTCTTCTTCTGTGAG \\
\hline CpopGR1q_fw & AGCTCCAGACAGTTCCTTTC \\
CpopGR1q_rev & AGATCTGGGTTGATGTCTTCGAG \\
\hline CpopOBP13q_fw & GCCAAGACCAAGAAGGGAGAAT \\
CpopOBP13q_rev & GATTTTGCCTTGTGCGTCCATG \\
\hline RNAi: & \\
\hline CpopLac2_rnai_fw & TAATACGACTCACTATAGGGAACGCTGGTACCCACTTCTG \\
CpopLac2_rnai_rev & TAATACGACTCACTATAGGGGTAACGCCACACTCTCCCAA \\
\hline PcoLac2_rnai_fw & TAATACGACTCACTATAGGGACGGCAAAGGACAATTCAGG \\
PcoLac2_rnai_rev & TAATACGACTCACTATAGGGAGGCCTGGGTTGCAAAATTC \\
\hline CpopTH_rnai_fw & TAATACGACTCACTATAGGGTCAACGCGCAGTATTGGTCT \\
CpopTH_rnai_rev & TAATACGACTCACTATAGGGGAACACCTTGCGGTACTCCA \\
\hline PcoTH_rnai_fw & TAATACGACTCACTATAGGGACAAGGAATACCGTGCCAGG \\
PcoTH_rnai_rev & TAATACGACTCACTATAGGGCCGAACTCGACAGTGAACCA \\
\hline RNA FISH: & \\
\hline CpopGR1_fish_fw & ATGTTCACTCGGATATTGGCGTT \\
CpopGR1_fish_rev & TCAGTTCCTAGGTATCTTCTGTATGTGA \\
\hline CpopOBP13_fish_fw & ATGTCTCCTTACAGTGCCAGTCCTGCAT \\
CpopOBP13_fish_rev & TTAAACAAGGCTGAGATGTGAGGGGG \\
\hline CpopActin_fish_fw & ATGTGTGACGATGATGTAGCGGC \\
CpopActin_fish_rev & TTAGAAGCACTTGCGGTGGACG \\
\hline & \\
\hline
\end{tabular}




\section{References:}

1 Lampasona, A. A. \& Czaplinski, K. RNA voyeurism: A coming of age story. Methods 98, 10-17 (2016). (doi:10.1016/j.ymeth.2015.11.024)

2 Richardson, D. S. \& Lichtman, J. W. Clarifying tissue clearing. Cell 162, 246-257 (2015). (doi:10.1016/j.cell.2015.06.067)

3 Zucker, R. M. Whole insect and mammalian embryo imaging with confocal microscopy: morphology and apoptosis. Cytometry. Part A 69, 1143-1152 (2006). (doi:10.1002/cyto.a.20343)

4 Susaki, E. A. \& Ueda, H. R. Whole-body and whole-organ clearing and imaging techniques with single-cell resolution: toward organism-level systems biology in mammals. Cell Chemical Biology 23, 137-157 (2016). (doi:10.1016/j.chembiol.2015.11.009)

5 Frétaud, M. et al. High-resolution 3D imaging of whole organ after clearing: taking a new look at the zebrafish testis. Scientific Reports 7, 43012 (2017). (doi:10.1038/Srep43012)

6 Susaki, E. A. et al. Whole-brain imaging with single-cell resolution using chemical cocktails and computational analysis. Cell 157, 726-739 (2014). (doi:10.1016/j.cell.2014.03.042)

7 Tainaka, K. et al. Whole-body imaging with single-cell resolution by tissue decolorization. Cell 159, 911-924 (2014). (doi:10.1016/j.cell.2014.10.034)

8 Li, W., Germain, R. N. \& Gerner, M. Y. Multiplex, quantitative cellular analysis in large tissue volumes with clearing-enhanced 3D microscopy (Ce3D). Proceedings of the National Academy of Sciences of the United States of America 114, E7321-E7330 (2017). (doi:10.1073/pnas.1708981114)

9 Susaki, E. A. et al. Advanced CUBIC protocols for whole-brain and whole-body clearing and imaging. Nature Protocols 10, 1709-1727 (2015).

10 Chung, K. et al. Structural and molecular interrogation of intact biological systems. Nature 497, 332-337 (2013). (doi:10.1038/nature12107)

11 Yang, B. et al. Single-cell phenotyping within transparent intact tissue through whole-body clearing. Cell 158, 945-958 (2014). (doi:10.1016/j.cell.2014.07.017)

12 Keller, P. J. \& Dodt, H.-U. Light sheet microscopy of living or cleared specimens. Current Opinion in Neurobiology 22, 138-143 (2012). (doi:10.1016/j.conb.2011.08.003)

13 Barek, H., Evans, J. \& Sugumaran, M. Unraveling complex molecular transformations of N- $\beta$ alanyldopamine that accounts for brown coloration of insect cuticle. Rapid Communications in Mass Spectrometry 31, 1363-1373 (2017). (doi:10.1002/rcm.7914)

14 Smolla, M., Ruchty, M., Nagel, M. \& Kleineidam, C. J. Clearing pigmented insect cuticle to investigate small insects' organs in situ using confocal laser-scanning microscopy (cLSM). Arthropod Structure \& Development 43, 175-181 (2014). (doi:10.1016/j.asd.2013.12.006)

15 Stöckl, A. L. \& Heinze, S. A clearer view of the insect brain - combining bleaching with standard whole-mount immunocytochemistry allows confocal imaging of pigment-covered brain areas for 3D reconstruction. Frontiers in Neuroanatomy 9 (2015). (doi:10.3389/fnana.2015.00121)

16 Koga, R., Tsuchida, T. \& Fukatsu, T. Quenching autofluorescence of insect tissues for in situ detection of endosymbionts. Applied Entomology and Zoology 44, 281-291 (2009). (doi:10.1303/aez.2009.281)

17 Kliot, A. \& Ghanim, M. Fluorescent in situ hybridization for the localization of viruses, bacteria and other microorganisms in insect and plant tissues. Methods 98, 74-81 (2016). (doi:10.1016/j.ymeth.2015.12.003)

18 Arakane, Y., Muthukrishnan, S., Beeman, R. W., Kanost, M. R. \& Kramer, K. J. Laccase 2 is the phenoloxidase gene required for beetle cuticle tanning. Proceedings of the National Academy of Sciences of the United States of America 102, 11337-11342 (2005). (doi:10.1073/pnas.0504982102)

19 Noh, M. Y., Muthukrishnan, S., Kramer, K. J. \& Arakane, Y. Cuticle formation and pigmentation in beetles. Current Opinion in Insect Science 17, 1-9 (2016). (doi:10.1016/j.cois.2016.05.004)

20 Dippel, S. et al. Morphological and transcriptomic analysis of a beetle chemosensory system reveals a gnathal olfactory center. BMC Biology 14, 90 (2016). (doi:10.1186/s12915-016-0304-z)

21 Dippel, S. et al. Tissue-specific transcriptomics, chromosomal localization, and phylogeny of chemosensory and odorant binding proteins from the red flour beetle Tribolium castaneum reveal subgroup specificities for olfaction or more general functions. BMC Genomics 15, 1141 (2014). (doi:10.1186/1471-2164-15-1141)

$22 \mathrm{Li}, \mathrm{X}$. M. et al. Candidate chemosensory genes identified in Colaphellus bowringi by antennal transcriptome analysis. BMC Genomics 16, 1028 (2015). (doi:10.1186/s12864-015-2236-3) 
Rabasović, M. D. et al. Nonlinear microscopy of chitin and chitinous structures: a case study of two cave-dwelling insects. Journal of Biomedical Optics 20, 016010 (2015). (doi:10.1117/1.JBO.20.1.016010)

24 Korytowski, W. \& Sarna, T. Bleaching of melanin pigments. Role of copper ions and hydrogen peroxide in autooxidation and photooxidation of synthetic dopa-melanin. Journal of Biological Chemistry 265, 12410-12416 (1990).

25 Alnuami, A. A., Zeedi, B., Qadri, S. M. \& Ashraf, S. S. Oxyradical-induced GFP damage and loss of fluorescence. International Journal of Biological Macromolecules 43, 182-186 (2008). (doi:10.1016/j.ijbiomac.2008.05.002)

26 Powell, M. E., Bradish, H. M., Gatehouse, J. A. \& Fitches, E. C. Systemic RNAi in the small hive beetle Aethina tumida Murray (Coleoptera: Nitidulidae), a serious pest of the European honey bee Apis mellifera. Pest Management Science 73, 53-63 (2017). (doi:10.1002/ps.4365)

27 Niu, B. L. et al. Cloning and RNAi-mediated functional characterization of MaLac2 of the pine sawyer, Monochamus alternatus. Insect Molecular Biology 17, 303-312 (2008). (doi:10.1111/j.1365-2583.2008.00803.x)

28 Gorman, M. J. \& Arakane, Y. Tyrosine hydroxylase is required for cuticle sclerotization and pigmentation in Tribolium castaneum. Insect Biochemistry and Molecular Biology 40, 267-273 (2010). (doi:10.1016/j.ibmb.2010.01.004)

29 Liu, C. et al. Repression of tyrosine hydroxylase is responsible for the sex-linked chocolate mutation of the silkworm, Bombyx mori. Proceedings of the National Academy of Sciences of the United States of America 107, 12980-12985 (2010). (doi:10.1073/pnas.1001725107)

30 Futahashi, R. et al. Laccase2 is required for cuticular pigmentation in stinkbugs. Insect Biochemistry and Molecular Biology 41, 191-196 (2011). (doi:10.1016/j.ibmb.2010.12.003)

31 Chen, E. H. et al. Tyrosine hydroxylase coordinates larval-pupal tanning and immunity in oriental fruit fly (Bactrocera dorsalis). Pest Management Science (2017). (doi:10.1002/ps.4738)

32 Elias-Neto, M., Soares, M. P., Simões, Z. L., Hartfelder, K. \& Bitondi, M. M. Developmental characterization, function and regulation of a Laccase2 encoding gene in the honey bee, Apis mellifera (Hymenoptera, Apinae). Insect Biochemistry and Molecular Biology 40, 241-251 (2010). (doi:10.1016/j.ibmb.2010.02.004)

33 Du, M.-H. et al. Suppression of Laccase 2 severely impairs cuticle tanning and pathogen resistance during the pupal metamorphosis of Anopheles sinensis (Diptera: Culicidae). Parasites \& Vectors 10, 171 (2017). (doi:10.1186/s13071-017-2118-4)

34 Okude, G. et al. Electroporation-mediated RNA interference reveals a role of the multicopper oxidase 2 gene in dragonfly cuticular pigmentation. Applied Entomology and Zoology 52, 379-387 (2017). (doi:10.1007/s13355-017-0489-9)

35 Strobl, F., Schmitz, A. \& Stelzer, E. H. K. Live imaging of Tribolium castaneum embryonic development using light-sheet-based fluorescence microscopy. Nature Protocols 10, 1486-1507 (2015). (doi:10.1038/nprot.2015.093)

36 Zimmerman, S. G., Peters, N. C., Altaras, A. E. \& Berg, C. A. Optimized RNA ISH, RNA FISH and protein-RNA double labeling (IF/FISH) in drosophila ovaries. Nature Protocols 8, 2158 (2013). (doi:10.1038/nprot.2013.136)

37 Barek, H., Sugumaran, M., Ito, S. \& Wakamatsu, K. Insect cuticular melanins are distinctly different from those of mammalian epidermal melanins. Pigment Cell \& Melanoma Research 31, 384-392 (2018). (doi:10.1111/pcmr.12672)

38 Anbutsu, H. et al. Small genome symbiont underlies cuticle hardness in beetles. Proceedings of the National Academy of Sciences of the United States of America 40, E8382-E8391 (2017). (doi:10.1073/pnas.1712857114)

39 Strauss, A. S. et al. Tissue-specific transcript profiling for $\mathrm{ABC}$ transporters in the sequestering larvae of the phytophagous leaf beetle Chrysomela populi. PLoS One 9, e98637 (2014). (doi:10.1371/journal.pone.0098637)

40 Stock, M. et al. Putative sugar transporters of the mustard leaf beetle Phaedon cochleariae: their phylogeny and role for nutrient supply in larval defensive glands. PLoS One 8, e84461 (2013). (doi:10.1371/journal.pone.0084461)

41 Livak, K. J. \& Schmittgen, T. D. Analysis of relative gene expression data using real-time quantitative PCR and the $2^{-(\mathrm{DD}}$ CT) method. Methods 25, 402-408 (2001). (doi:10.1006/meth.2001.1262) 